\title{
Phenolic complex of red wine materials from grapes growing in the Crimea
}

\author{
Dmitry Yermolin ${ }^{1,}$, , Galina Yermolina ${ }^{1}$, Yuriy Gerber $^{1}$, Daria Zadorozhnaya ${ }^{1}$, Zinaida \\ Kotolovets $^{2}$ \\ ${ }^{1}$ V.I. Vernadskiy Crimean Federal University, 4, Prospekt Vernadskogo, Simferopol, Republic of \\ Crimea, Russia \\ ${ }^{2}$ Magarach All-Russia National Research Institute for Viticulture and Wine-Making, 31, str. Kirov, \\ Yalta, Republic of Crimea, Russia
}

\begin{abstract}
The research work presents the results of studies of the composition of anthocyanins, monomeric forms of phenolic substances, oligomeric procyanidins, polymer forms of phenolic substances in wine materials Cabernet Sauvignon, Merlot, Syrah, Petit Verdot, Sangiovese, Marcelan obtained from grapes growing in the Crimea. It was found that the main coloring material in the wine materials under study was malvidin3 -O-glycoside. The proportion of this compound ranged from $42.9 \%$ to $54.3 \%$ in the total of all anthocyanins. The highest mass concentrations of oxybenzoic and hydroxycinnamic acids are determined in the wine material Marcelan. In the Sangiovese wine material, the highest concentrations of flavan-3-ols are determined. Mass concentrations of quercetin-3-O-glucoside in the test samples were $4-17 \mathrm{mg} / \mathrm{dm}^{3}$; quercetin $1-7 \mathrm{mg} / \mathrm{dm}^{3}$. Mass concentrations of oligomeric procyanidins were in the range of $106-240 \mathrm{mg} / \mathrm{dm}^{3}$ and the highest value was determined in the wine material Marcelan, the lowest - Cabernet Sauvignon. Mass concentrations of polymer forms of phenolic substances amounted to 1487$2998 \mathrm{mg} / \mathrm{dm}^{3}$.
\end{abstract}

\section{Introduction}

The main anthocyanins of Vitis vinifera grapes are monoglycosides of malvidin (more than 50\%), peonidine, dolphinidin, petunidin, in a smaller amount - cyanidin and acylated monoglycosides of peonidine and maldivine [1-6].

Vitis vinifera anthocyanins are 3-O-monoglycosides and 3-O-acylated monoglycosides of the five main anthocyanoidins - dolphidine, cyanidin, petunidin, peonidine and malvidin - which differ in number and location of the hydroxyl or methoxy group of the B-ring of the molecule.

Malvidin is the main anthocyanin of red grape varieties (up to $90 \%$ in Grenache and up to $50 \%$ in Sangiovese). The number of acylated anthocyanins is very dependent on the variety.

*Corresponding author: dimayermolin@mail.ru 
The main monomorphic forms of phenolic substances in wines are hydroxybenzoic acids, hydroxycinnamic acids, flavan-3-ols and flavanols.

Hydroxybenzoic acids are derivatives of benzoic acid and have a C6-C1 skeleton. In total, seven derivatives of benzoic acid were found in wine. Most often they are in free forms. Gallic acid is the largest hydroxybenzoic acid in wine [7].

Hydroxycinnamic acids have a C6-C3 skeleton; their main representatives are pcoumaric and ferulic acids. Free hydroxycinnamic acids are rare in wine, their esters with tartaric acid predominate [8]. Kaftaric acid dominates among them (up to 50\% of all hydroxycinnamic acids).

Five catechins were found in wine: (-) - epigallocatechin, (+) - gallocatechin, (-) catechin, (+) -catechin and (-) -epicatechin [9.10]. During the oxidation of catechins, they polymerize to form condensed tannins. Flovan-3-ol show the ability to increase the color intensity of anthocyanins.

An important property of flavonols (quercetin, kempferol, myricetin and their glycosides) is participation in redox processes [11-15].

It should be noted that the data on the composition of the phenolic complex of wine materials and Crimean wines are very limited. In this regard, the aim of this work is to study the complex of anthocyanins, hydroxybenzoic acids, hydroxycinnamic acids, flavan3-ol, flavonols, procyanidins, and polymeric forms of phenolic substances in red wine materials produced in Crimea.

\section{Materials and methods}

In the paper, the methods of analysis of wine materials generally accepted in wine chemistry were used.

The mass concentration of phenolic and coloring substances was determined using an Agilent Technologies chromatograph (model 1100) equipped with a G1379A flow-through vacuum degasser, 4-channel low-pressure gradient pump G13111A, G1313A automatic injector, G13116A column thermostat, and G1316A diode array detector. For carrying out the analysis, a chromatographic column $2.1 \times 150 \mathrm{~mm}$ in size, filled with an octadecylsilyl sorbent, grain size $3.5 \mu \mathrm{m}$, ZORBAX-SB C-18 was used.

For carrying out the analysis, the following chromatography mode is set:

- feed rate of the mobile phase $0.25 \mathrm{ml} / \mathrm{min}$;

- gradient chromatographic mode is presented in Table 1.

Table 1. Gradient chromatography mode.

\begin{tabular}{|c|c|c|c|}
\hline Time min & A\% (0.6\% TFA*) & $\begin{array}{c}\text { B\% 70\% - methanol } \\
(\mathbf{0 . 6 \%} \text { TFA })\end{array}$ & $\begin{array}{c}\text { C\% 100 \% } \\
\text { methanol }\end{array}$ \\
\hline 0 & 92 & 8 & 0 \\
\hline 8 & 62 & 38 & 0 \\
\hline 24 & 0 & 100 & 0 \\
\hline 24.1 & 0 & 0 & 100 \\
\hline 29 & 0 & 0 & 100 \\
\hline
\end{tabular}

The detection parameters are set as follows:

- measurement scale 1.0;

- scan time $0.5 \mathrm{sec}$.

- spectrum acquisition parameters - each peak is 190-600 nm

Phenolic was identified by retention times of standards and spectral characteristics.

Previously, wine materials were centrifuged.

The mass concentration of the polymer forms of phenolic substances was determined by the colorimetric method using the Folin - Chocalteu reagent. 
The research materials were wine materials from the varieties Cabernet-Sauvignon, Merlot, Syrah, Petit Verdot, Sangiovese and Marcelan, developed in the Crimea.

The grapes were processed according to the red method, the scheme "pulp fermentation" was used. Wort yield was $60 \mathrm{dal} / \mathrm{t}$.

\section{Results and discussions}

At the first stage of the study, the composition of anthocyanins in the experimental wine materials was studied. Moreover, it was found that in the wine material CabernetSauvignon, the main coloring matter is malvidin-3-O-glycoside (more than 50\% in the sum of all anthocyanins). Significant concentrations also contain malvidin-3-O- (6'-acetylglucoside) (19.9\%), delphinidin-3-O-glycoside (6.5\%), petunidin-3-O-glucoside $(6,3 \%)$, malvidin-3-O- (6'-p-coumaroyl-glucoside) (4.4\%), delphinidin-3-O- (6'-acetyl-glucoside) $(3.5 \%)$, peonidine- 3-O-glucoside $(2.7 \%)$. The proportion of the remaining specific anthocyanins did not exceed $2 \%$ (Figure 1).

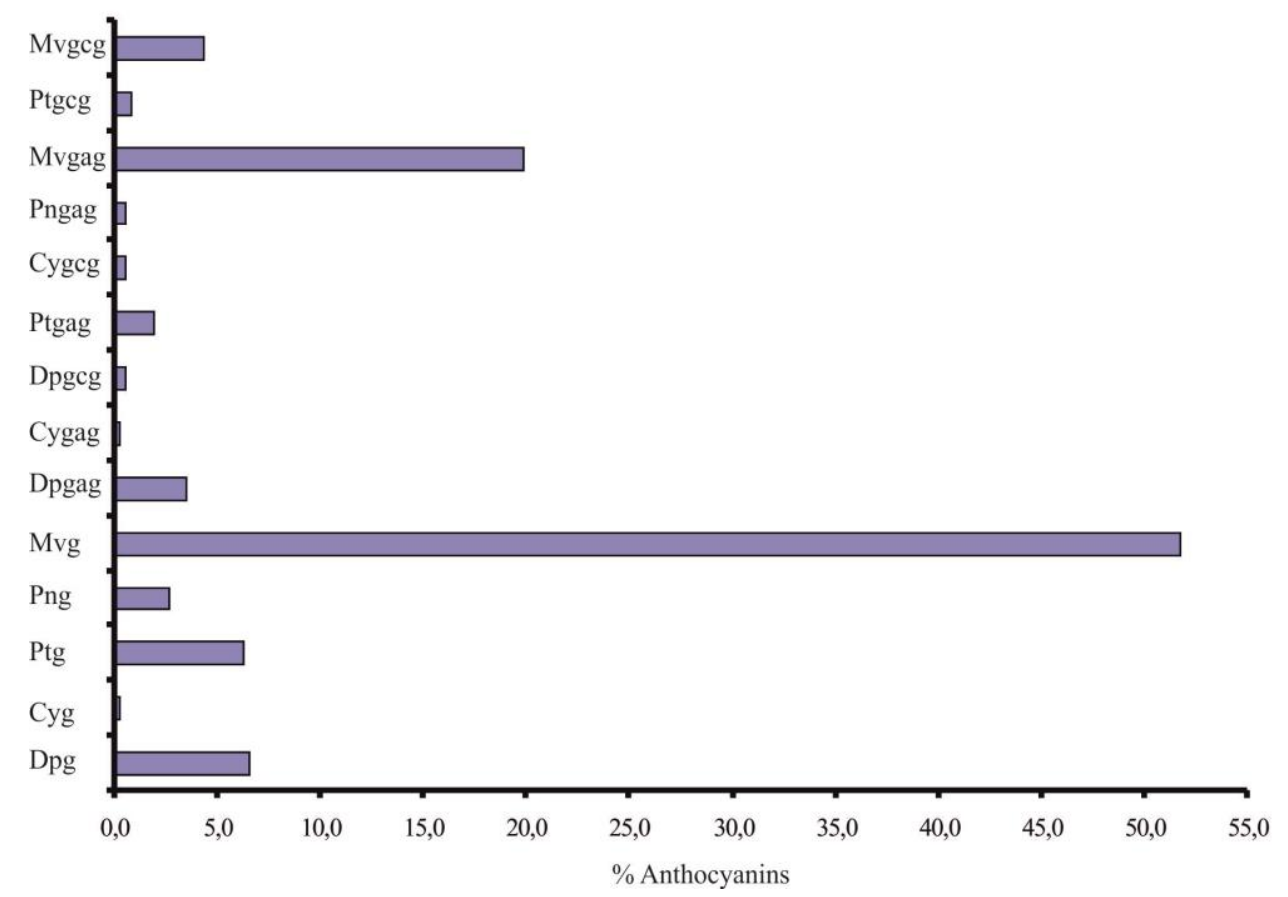

Fig. 1. The composition of the anthocyanins of the wine material Cabernet Sauvignon.

Where: Dpg=delphinidin-3-glucoside, Cyg=cyanidin-3-glucoside, Ptg=petunidin-3glucoside, Png=peonidin-3-glucoside, Mvg=malvidin-3-glucoside, Dpgag= Delphinidin-3O-(6'-acetyl-glycoside), Cygag=Cyanidin-3-O-(6'-acetyl-glycoside), Dpgcg=Delphinidin-3O-(6'-p-coumaroyl-glycoside), Ptgag=Petunidin-3-O-(6'-acetyl-glycoside), Cygcg=Cyanidin-3-O--(6'-p-coumaroyl-glycoside), Pngag=Peonidin-3-O-(6'-acetylglycoside), Mvgag=Malvidin-3-O-(6'-acetyl-glycoside), Ptgcg=Petunidin-3-O-(6'-pcoumaroyl-glycoside), Mvgcg= Malvidin-3-O-(6'-p-coumaroyl-glycoside).

In the Merlot wine material, the proportion of malvidin-3-O-glycoside was $50 \%$ of all certain anthocyanins. The proportion of malvidin-3-O- (6'-acetyl-glucoside) was $14.3 \%$, atunidin-3-O-glucoside - 7.1\%, peonidin-3-O-glucoside - 7.1\%, malvidin-3 -O- (6'-pcoumaroyl-glucoside) - 5.8\%, delphinidin-3-O-glycoside - 5.2\%, delphinidin-3-O- (6'- 
acetyl-glucoside) - $3.2 \%$ As for the Cabernet-Sauvignon wine material, the share of other anthocyanins did not exceed $2 \%$ (Figure 2).

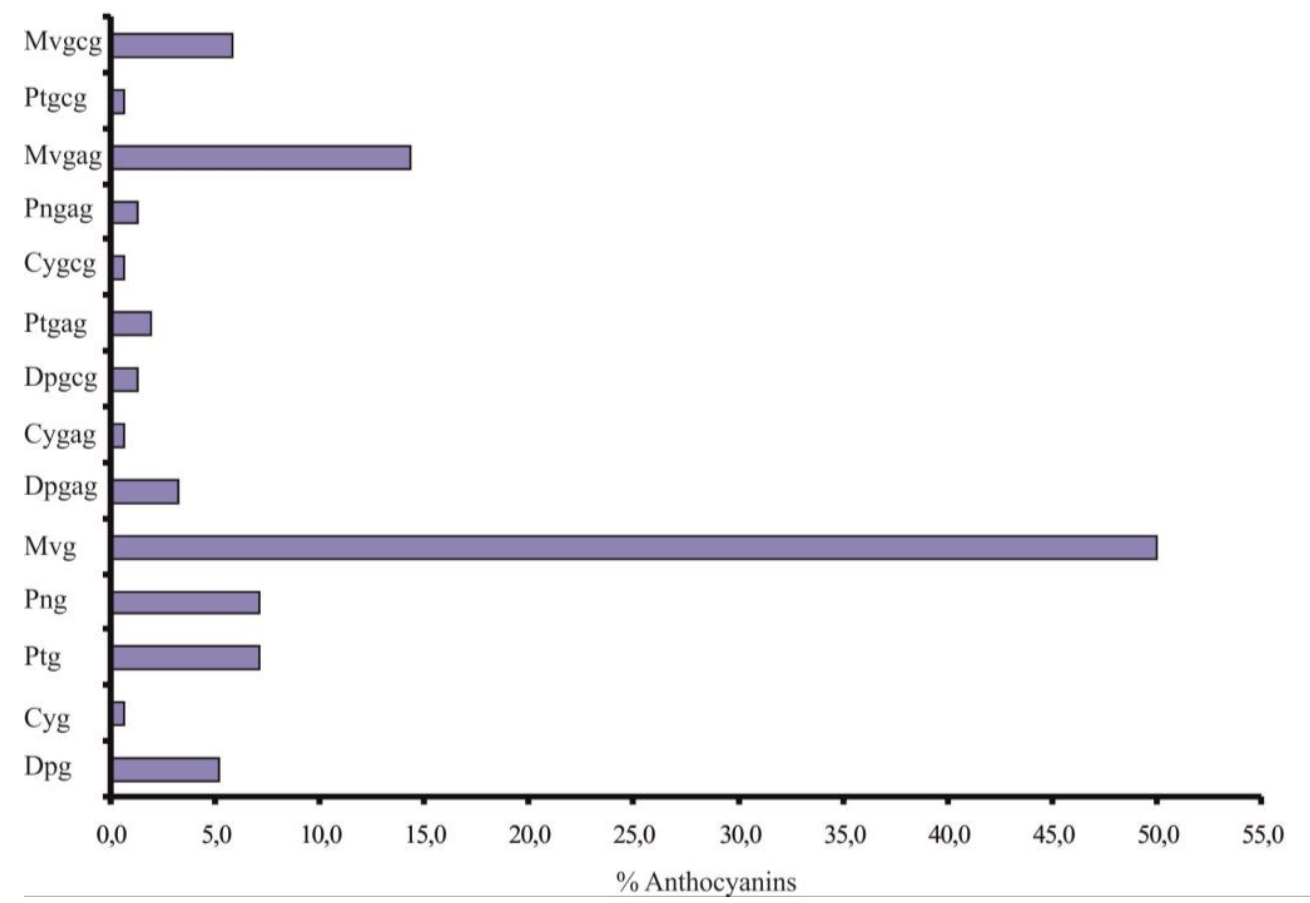

Fig. 2. The composition of the anthocyanins Merlot wine material.

When studying the complex of anthocyanins of the Syrah wine material, it was found that the proportion of malvidin-3-O-glycoside was $52.1 \%$, malvidin-3-O- (6'-acetylglucoside) - 16.3\%, petunidin-3-O -glucoside - 7.9\%, malvidin-3-O- (6'-p-coumaroylglucoside) - 5.3\%, delphinidin-3-O-glycoside - 4.7\%, peonidin-3-O-glucoside and delphinidin-3-O- (6'-acetyl-glucoside), 3.4\% each.

A distinctive feature of the wine material Petit Verdot was a relatively high concentration of delphinidin-3-O-glycoside (10.9\%), petunidin-3-O-glucoside (9\%). The proportion of malvidin-3-O-glycoside was $42.9 \%$, which is significantly less than in all other samples. Malvidin-3-O- (6'-acetyl-glucoside) was also determined in a significant concentration $-16.8 \%$ of all certain anthocyanins (Figure 4 ).

It was characteristic of the wine material from the Sangiovese variety that the proportion of malvidin-3-O-glycoside was at the level of $50.2 \%$ of all the determined anthocyanins. A distinctive feature of this wine material was that the proportion of dolphinidin-3-O- (6'-acetyl-glucoside) exceeded this indicator in all other samples studied and amounted to $9.0 \%$ (Figure 5). 


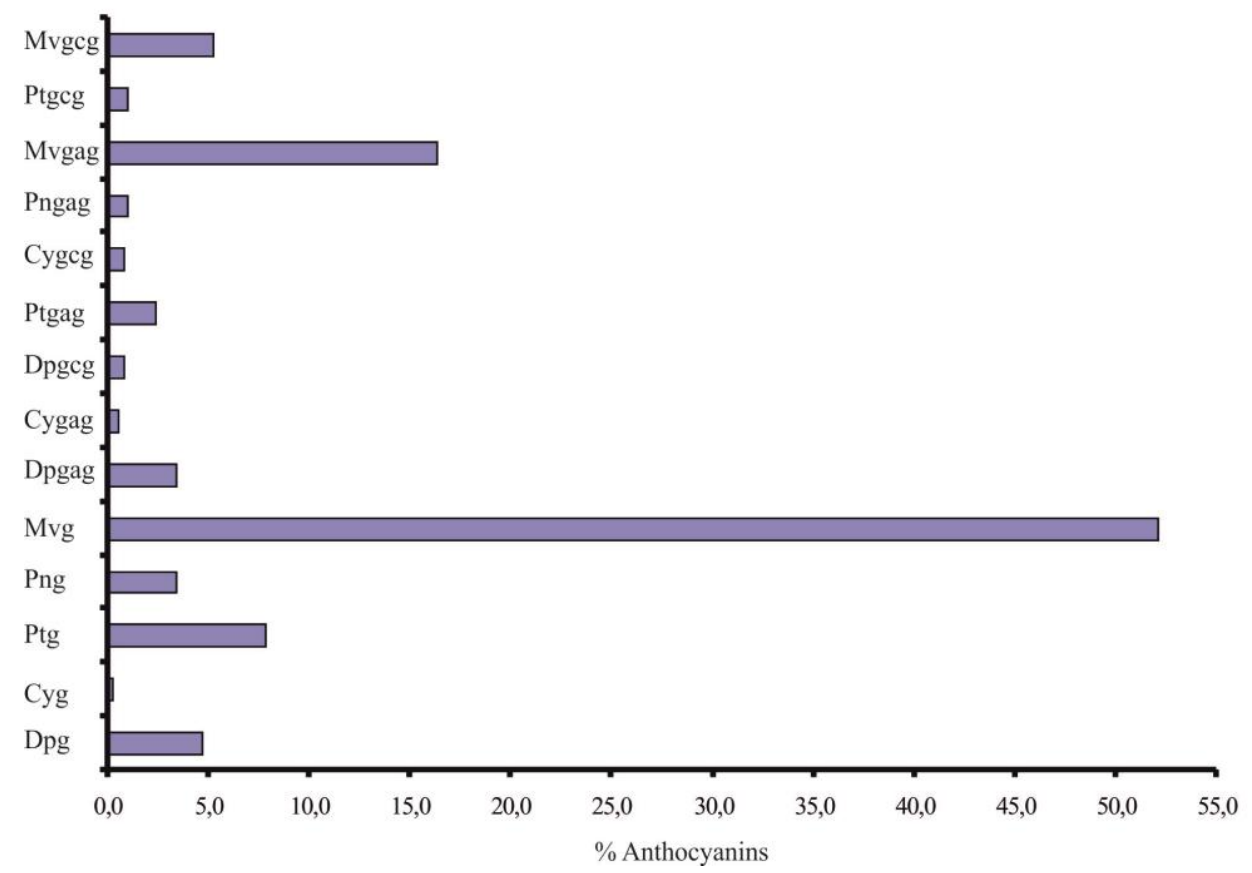

Fig. 3. Composition of anthocyanins of Syrah wine material.

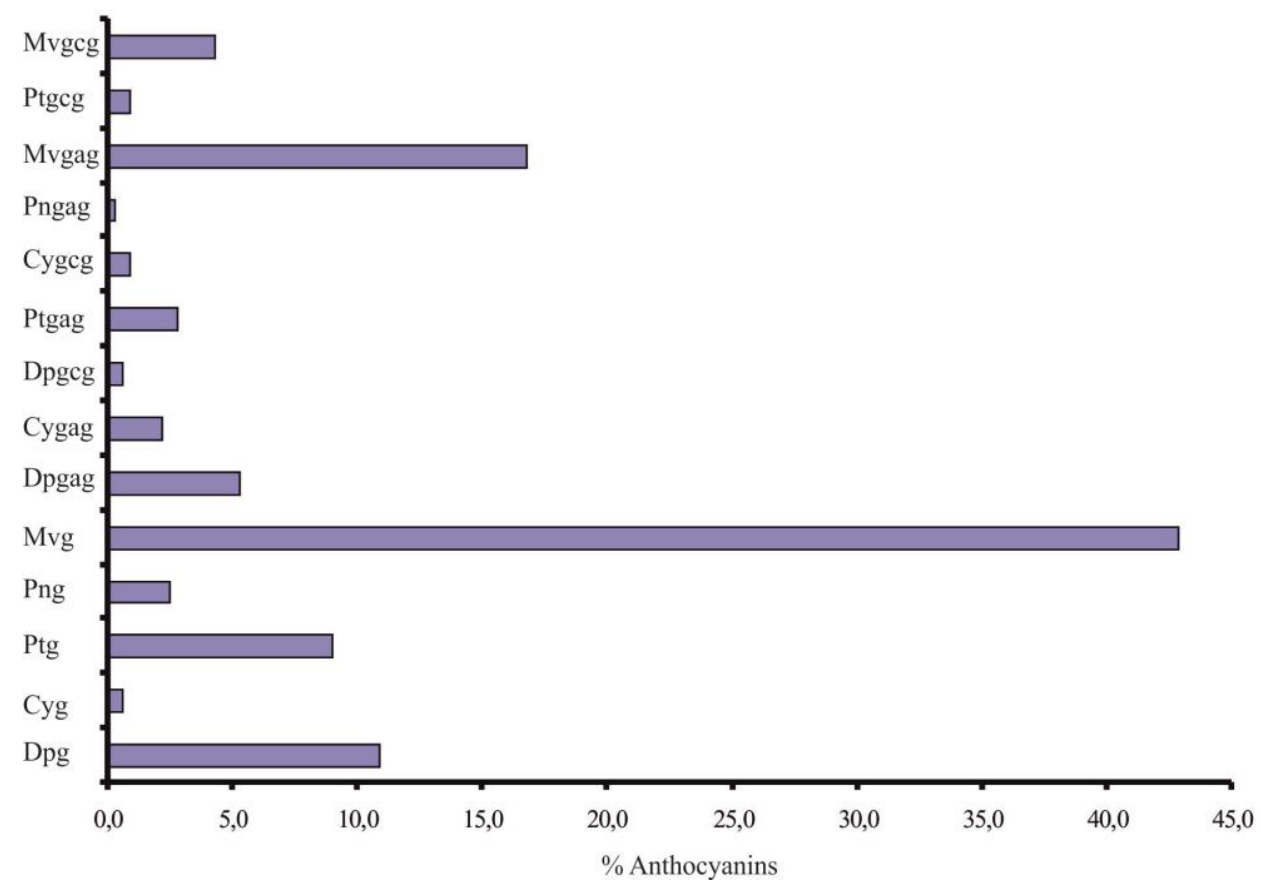

Fig. 4. Composition of anthocyanins of wine material Petit Verdot. 


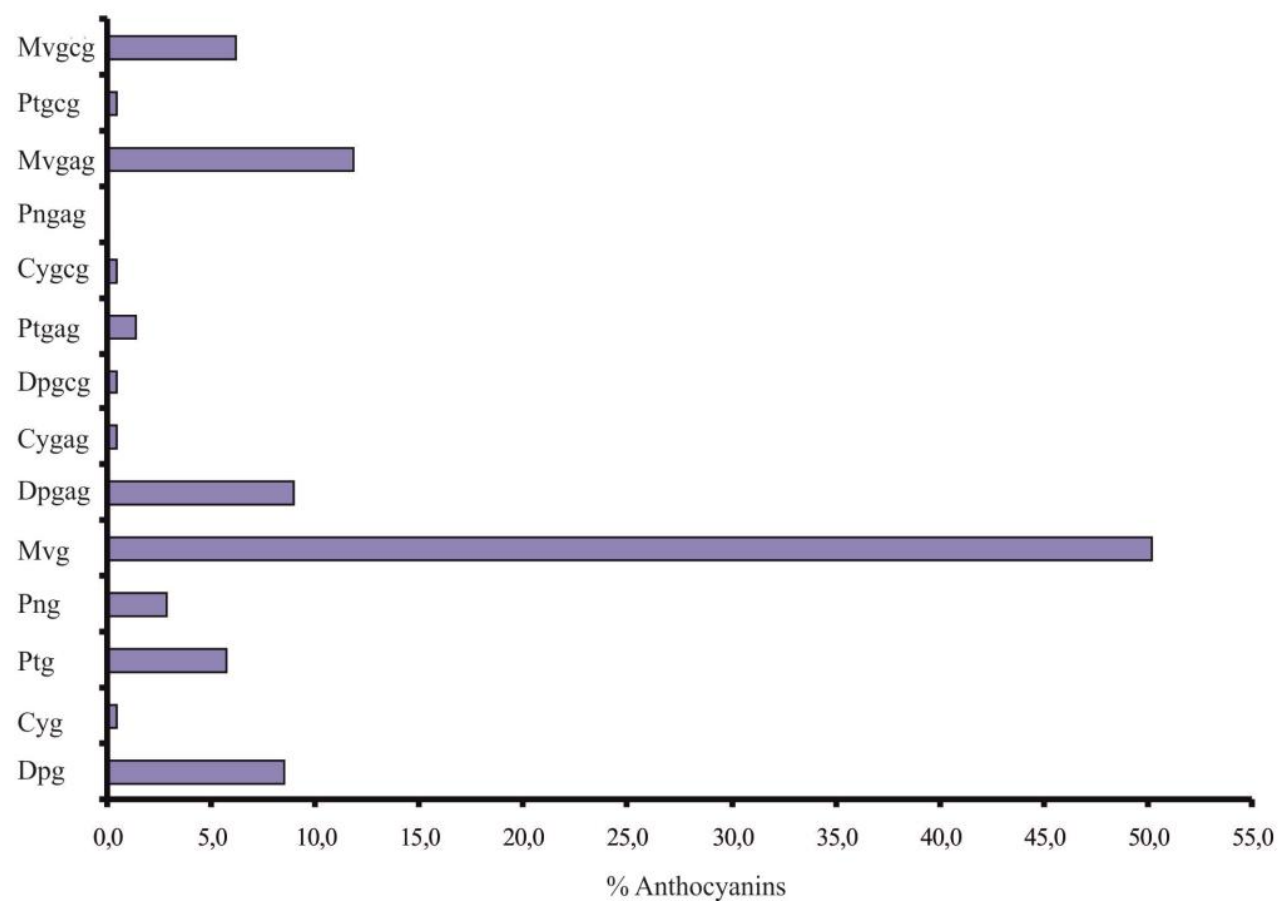

Fig. 5. Composition of anthocyanins of Sangiovese wine material.

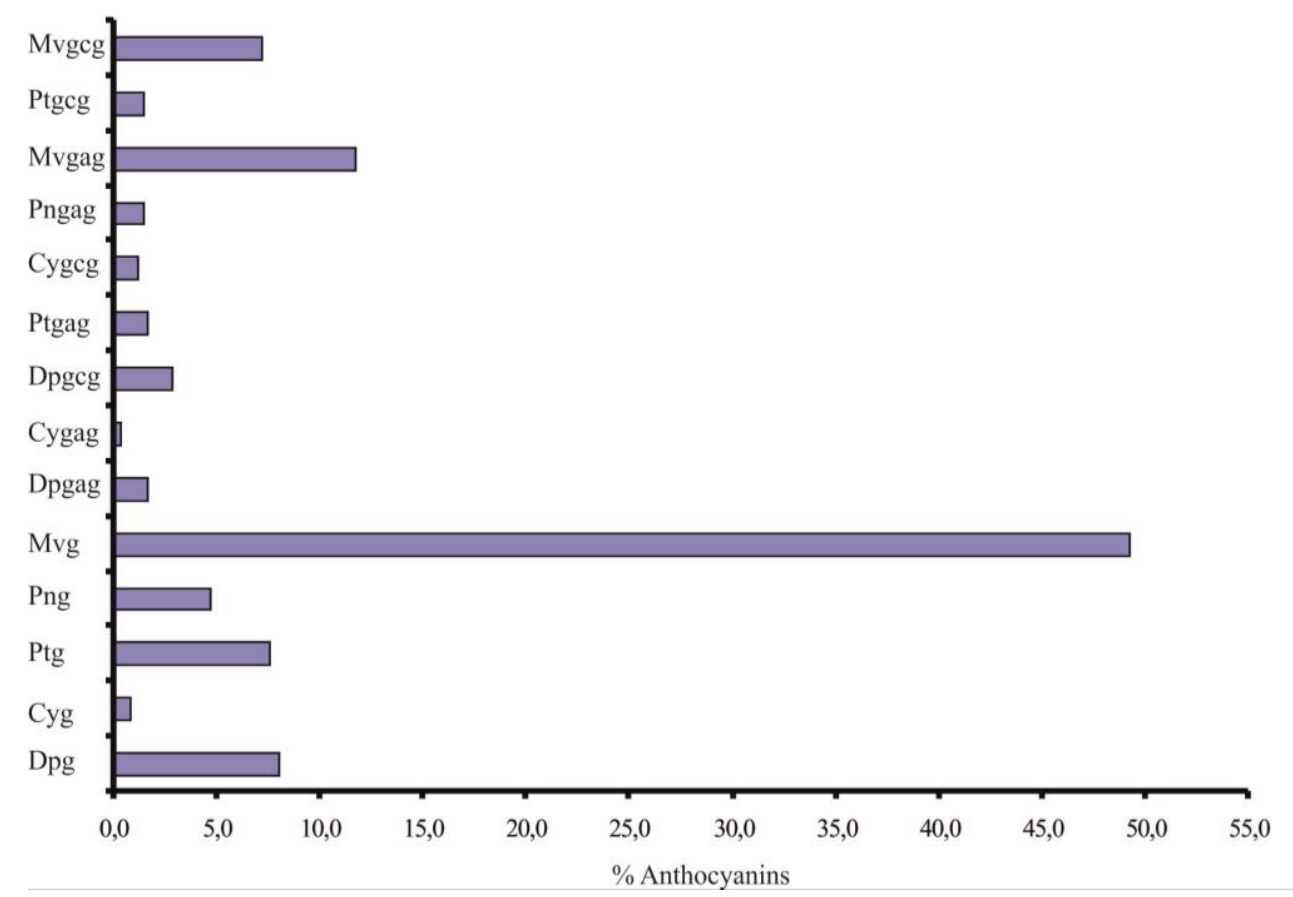

Fig. 6. The composition of the anthocyanins of the wine material Marcelan.

A study of the composition of the complex of anthocyanins of Marcelan wine material showed that a distinctive feature of this variety is the relatively high concentration of malvidin-3-O- (6'-p-coumaroyl-glucoside) and amounted to $7.2 \%$ in the sum of all the 
determined anthocyanins and a low concentration of malvidin-3 -O- (6'-acetyl glucoside) $11.7 \%$ (which is also characteristic of the Sangiovese variety). The proportion of malvidin3-O-glucoside was $49.3 \%$. The following anthocyanins were also determined in significant concentrations: dolphinidin-3-O-glycoside $8.0 \%$, petunidin-3-O-glucoside $7.6 \%$, peonidin3-O-glucoside 4.7\%, delphinidin-3-O- (6'-p-coumaroyl-glucoside) 2.9\% (Figure 6).

At the next stage of studies, mass concentrations of monomeric phenolic substances were determined. The data obtained are presented in table 2 .

Table 2. Mass concentrations of monomeric forms of phenolic substances in the studied wine materials, $\mathrm{mg} / \mathrm{dm}^{3}$.

\begin{tabular}{|l|c|c|c|c|c|c|}
\hline $\begin{array}{c}\text { Phenolic } \\
\text { substances }\end{array}$ & $\begin{array}{c}\text { Cabernet } \\
\text { Sauvignon }\end{array}$ & Merlot & Syrah & $\begin{array}{c}\text { Petit } \\
\text { Verdot }\end{array}$ & Sangiovese & Marcelan \\
\hline \multicolumn{7}{|c|}{ Hydroxybenzoic acid } \\
\hline Gallic acid & 12 & 27 & 20 & 29 & 33 & 48 \\
\hline Lilac acid & 5 & 2 & 4 & 7 & 4 & 3 \\
\hline Flovan-3-ols & \multicolumn{7}{|c|}{ Hydroxycinnamic Acids } \\
\hline (+)-D- Catechin & 26 & 39 & 46 & 50 & 119 & 78 \\
\hline (-)-Epicatechin & 27 & 52 & 74 & 44 & 111 & 56 \\
\hline \multicolumn{7}{|c|}{ Flavonols } \\
\hline Kaftaric acid & 23 & 17 & 30 & 17 & 36 & 104 \\
\hline Cautaric acid & 2 & 2 & 6 & 2 & 4 & 45 \\
\hline \multicolumn{7}{|c|}{} \\
\hline $\begin{array}{l}\text { Quercetin-3-O- } \\
\text { Glucoside }\end{array}$ & 5 & 5 & 8 & 4 & 4 & 17 \\
\hline Quercetin & 1 & 1 & 2 & 4 & 1 & 7 \\
\hline Total & 101 & 145 & 190 & 157 & 312 & 358 \\
\hline
\end{tabular}

Analysis of the data presented in table 2 indicates that the highest mass concentration of monomeric forms of phenolic substances was determined in the wine materials Marcelan and Sangiovese, the smallest - Cabernet-Sauvignon. The mass concentration of gallic acid in the highest concentration is determined in the wine material Marcelan, lilac acid - Petit verdot. In the Sangiovese wine material, the highest mass concentrations of flavan-3-ols are determined. Mass concentrations of hydroxycinnamic acids in the highest concentration were recorded in Marcelan wine material. Mass concentrations of quercetin-3-O-glucoside in the studied materials were $4-17 \mathrm{mg} / \mathrm{dm}^{3}$; quercetin $-1-7 \mathrm{mg} / \mathrm{dm}^{3}$.

In the samples under study, mass concentrations of oligomeric procyanidins and polymer forms of phenolic substances were determined. The data obtained are shown in table 3.

Table 3. Mass concentrations of oligomeric and polymeric forms of phenolic substances, $\mathrm{mg} / \mathrm{dm}^{3}$.

\begin{tabular}{|l|c|c|c|l|l|l|}
\hline \multicolumn{1}{|c|}{ Phenolic substances } & $\begin{array}{c}\text { Cabernet } \\
\text { Sauvignon }\end{array}$ & $\begin{array}{l}\text { Merl } \\
\text { ot }\end{array}$ & $\begin{array}{l}\text { Syra } \\
\text { h }\end{array}$ & $\begin{array}{l}\text { Petit } \\
\text { Verd } \\
\text { ot }\end{array}$ & $\begin{array}{l}\text { Sangiov } \\
\text { ese }\end{array}$ & $\begin{array}{l}\text { Marcel } \\
\text { an }\end{array}$ \\
\hline Oligomeric Procyanidins & 106 & 178 & 175 & 181 & 185 & 240 \\
\hline $\begin{array}{l}\text { Polymer forms of phenolic } \\
\text { substances }\end{array}$ & 1712 & 1487 & 2022 & 1826 & 1292 & 2998 \\
\hline
\end{tabular}

Mass concentrations of oligomeric procyanidins were in the range of $106-240 \mathrm{mg} / \mathrm{dm}^{3}$ and the highest value was determined in the wine material Marcelan, the lowest - CabernetSauvignon (table 3). Mass concentrations of polymer forms of phenolic substances amounted to $1292-2998 \mathrm{mg} / \mathrm{dm}^{3}$. 


\section{Conclusions}

As a result of the studies, it was found that the main coloring matter in the studied wine materials was malvidin-3-O-glycoside. The proportion of this compound ranged from $42.9 \%$ to $54.3 \%$ in the total of all anthocyanins. Delphinidin-3-O-glycoside, petunidin-3-Oglucoside, malvidin-3-O- (6'-acetyl-glucoside), malvidin-3-O- (6'-p-coumaroyl- glucoside) The highest mass concentrations of hydroxybenzoic and hydroxycinnamic acids are determined in the wine material Marcelan. In the Sangiovese wine material, the highest concentrations of flavan-3-ols are determined. Mass concentrations of quercetin-3-Oglucoside in the test samples were $4-17 \mathrm{mg} / \mathrm{dm}^{3}$; quercetin - 1-7 $\mathrm{mg} / \mathrm{dm}^{3}$. Mass concentrations of oligomeric procyanidins were in the range of $106-240 \mathrm{mg} / \mathrm{dm}^{3}$ and the greatest value was determined in the wine material Marcelan, the smallest - CabernetSauvignon. Mass concentrations of polymer forms of phenolic substances amounted to $1292-2998 \mathrm{mg} / \mathrm{dm}^{3}$.

\section{References}

1. J. Balík, M. Kumšta, O. Rop, Chemical Papers 67, 1285-1292 (2013) doi: 10.2478/s11696-013-0378-9

2. Ke Zhang, Lin Yuan, Qian Li, Rui Wang, Zhen-Zhen Zhang, OENO One 53 (2019) doi: https://doi.org/10.20870/oeno-one.2019.53.3.2460

3. D. Yermolin, D. Zadorozhnaya, G. Yermolina, V. Ivanchenko, P. Dogoda, IOP Conf. Series: Earth and Environmental Science 403 (2019) doi:10.1088/1755$1315 / 403 / 1 / 012070$

4. K. Bindon, S. Kassara, Y. Hayasaka, A. Schulkin, P. Smith, J. Agric. Food Chem. 62, 11582-1159 (2014) doi: 10.1021/jf503922h

5. M. Figueiredo-González, E. Martinez-Carballo, B. Cancho-Grande, J.L. Santiago, M.C. Martinez, J. Simal-Gándara, Food Chemistry 130, 9-19 (2012) doi: 10.1016/j.foodchem.2011.06.006

6. K. Fraige, E.R. Pereira-Filho, E. Carrilho, Food Chemistry 145, 395-403 (2014) doi: 10.1016/j.foodchem.2013.08.066

7. X. Zhang, F. He, B. Zhang, M.J. Reeves, Y. Liu, X. Zhao, C. Duan, Food Research International 106, 568-579 (2018) doi: 10.1016/j.foodres.2017.12.054

8. H. Kalkan Yıldııım, A. Altındışli, Journal International Journal of Food Properties 18 (2015)

9. G. Parpinello, A. Rombolà, M. Simoni, A. Versari, Food Chemistry 167, 145-152 (2015)

10. V. Ivanova, M. Stefova, B. Vojnoski, Á. Dörnyeid, L. Márk, V. Dimovska, T. Stafilov, F. Kilár, Food Research International 44, 2851-2860 (2011) doi: 10.1016/j.foodres.2011.06.046

11. F. Dias, J. David, J. David, J. Braz. Chem. Soc. 27, 1055-1059 (2016)

12. Ran-Ran Xing, Fei He and others, S. Afr. J. Enol. Vitic. 36, 32-43 (2015)

13. Si-Yu Li, Fei He, Bao-Qing Zhu, Jun Wang, Chang-Qing Duan, International Journal of Food Properties 20 (2017) doi:10.1080/10942912.2016.1233117

14. D. Yermolin, D. Zadorozhnaya, Winemaking and viticulture 3, 22-24 (2015)

15. I. Hermosín-Gutiérrez, G. Favre, G. Gonzalez-Neves, D. Piccardo, Food Research International 112 (2018) doi: 10.1016/j.foodres.2018.06.019 